Meta

Journal des traducteurs

Translators' Journal

\title{
Autotraducciones: Una perspectiva histórica
}

\section{Julio César Santoyo}

Volume 50, numéro 3, août 2005

Le prisme de l'histoire

The History Lens

URI : https://id.erudit.org/iderudit/011601ar

DOI : https://doi.org/10.7202/011601ar

Aller au sommaire du numéro

Éditeur(s)

Les Presses de l'Université de Montréal

ISSN

0026-0452 (imprimé)

1492-1421 (numérique)

Découvrir la revue

Citer cet article

Santoyo, J. C. (2005). Autotraducciones: Una perspectiva histórica. Meta, 50(3), 858-867. https://doi.org/10.7202/011601ar

\section{Résumé de l'article}

Malgré l'opinion presque unanime selon laquelle « les exemples d'autotraduction sont rarissimes ", une longue tradition d'autotraductions jalonne l'histoire du dernier millénaire. À ce propos, citons Tho. More, Du Bellay, Calvin, John Donne, Goldoni, Mistral, Tagore, Beckett, Aitmatov ou Julien Green. De plus en plus fréquent, ce phénomène mérite une analyse bien plus approfondie que celles qui lui ont été consacrées jusqu’à présent.
Ce document est protégé par la loi sur le droit d'auteur. L'utilisation des services d’Érudit (y compris la reproduction) est assujettie à sa politique d'utilisation que vous pouvez consulter en ligne.

https://apropos.erudit.org/fr/usagers/politique-dutilisation/ 


\title{
Autotraducciones: Una perspectiva histórica
}

\author{
JULIO CÉSAR SANTOYO \\ Universidad de León, León, España \\ dfmjsm@unileon.es
}

\begin{abstract}
RÉSUMÉ
Malgré l'opinion presque unanime selon laquelle «les exemples d'autotraduction sont rarissimes», une longue tradition d'autotraductions jalonne l'histoire du dernier millénaire. À ce propos, citons Tho. More, Du Bellay, Calvin, John Donne, Goldoni, Mistral, Tagore, Beckett, Aitmatov ou Julien Green. De plus en plus fréquent, ce phénomène mérite une analyse bien plus approfondie que celles qui lui ont été consacrées jusqu'à présent.
\end{abstract}

\begin{abstract}
In spite of today's almost unanimous opinion on the subject ("Historically, few authors have dared to translate their own works"), a long tradition of self-translations runs through the history of the last millennium. It is enough to think of Tho. More, Du Bellay, Calvin, John Donne, Goldoni, Mistral, Tagore, Beckett, Aitmatov or Julian Green. A cultural and linguistic phenomenon which is increasingly frequent, it deserves much more attention than it has been received to this date.
\end{abstract}

\section{MOTS-CLÉS/KEYWORDS}

perspective historique, autotraduction, traduction d'auteur

Indeed, self-translation is a much more widespread phenomenon than one might think

(Whyte 2002: 64)

There is no more intimate or problematic relationship between translator and author than what occurs in self-translation

(Wechsler 1998: 213)

Traducción de autor, autotraducción, self-translation, Selbstübersetzung, autotraduzione... Hace ya casi treinta años que Anton Popovic la definió como "the translation of an original work into another language by the author himself” (1976: 19). A este respecto, resulta sorprendente lo erróneos que pueden llegar a ser determinados tópicos.

Richard S. Sylvester, de la Universidad de Yale, escribía en 1963: "It is rare enough... for an author to compose a work in one language and then translate it into another". Subrayo: Rare enough.

En 1984, en L'épreuve de l'étranger, Antoine Berman insistía: "Pour nous, les autotraductions sont des exceptions". Subrayo: Exceptions.

En noviembre de 1999 Grady Miller repetía (1999: 11): "Historically, few authors have dared to translate their own works". Subrayo: Few authors.

A su vez, el escritor y profesor de la Universidad de Nueva York Raymond Federman comentaba hace unos años, en la misma línea de pensamiento (1993: 76): "I also, at times, translate my own work either from English into French or vice versa. 
That self-translating activity is certainly not very common in the field of creative writing. In that sense then, I am somewhat of a phenomenon. The French would say: Federman, c'est un drôle de phénomène!'.

Opinión todavía más reciente es la de Christian Balliu, profesor en el Institut Supérieur de Traducteurs et Interprètes, en Bruselas, cuando hace tres años escribía en esta misma revista (2001: 99): “On en conviendra, les exemples d'autotraduction... sont rarissimes dans le domaine littéraire et ne font qu'exception, comme Nabokov et son Lolita, pour citer un cas..." Vuelvo a subrayar: Rarissimes.

Hace cuatro años que John Benjamins publicó un volumen, en edición de Allison Beeby \& al., con el título de Investigating Translation; en una de los artículos allí incluidos, Helena Tanqueiro (2000: 50) informa al lector de que "while it is true that throughout history there have been many writers who wrote in more than one language, such as for example, Paul Celan, Derek Walcott, Samuel Beckett, Primo Levi, Jorge Semprún, Antonio Tabucci, it is nevertheless interesting to see that only a few, very few indeed, actually translated their own work, despite the fact that all of these, and other important writers such as Hölderlin, Ezra Pound, Valéry, and so on, devoted much of their lives to translation..." Subrayo nuevamente: Few, very few indeed.

Tantos son los testimonios, todos en el mismo sentido y dirección, que tal parece ser hoy 'el estado de la cuestión'. Y cuando tantas opiniones, y tan cualificadas, y a lo largo de los últimos cuarenta años, coinciden en los términos en que lo hacen..., uno no puede dejar de pensar que todas ellas han de estar en lo cierto.

Nada extraño resulta, pues, que la autotraducción esté hoy considerada como algo absolutamente marginal, una especie de rareza cultural o literaria, residuo menor, rincón oscuro y apartado quizá de la Literatura Comparada, tal vez de los Estudios de Traducción, acaso de la Lingüística Contrastiva. Nada extraño resulta tampoco, por lo mismo, que Brian T. Fitch, escribiera en su estudio Beckett and Babel (1988: 21) "Direct discussion or even mention of self-translation is virtually non-existent in writings on theory of translation". O que nada menos que el Dictionary of Translation Studies, de Shuttleworth \& Cowie (1997: 13), afirme categóricamente: "Little work has been done on autotranslation".

La realidad, sin embargo, es muy otra. Porque lo cierto es que la autotraducción no es ni siquiera "característica de la particular configuración lingüística y cultural de la Europa del Renacimiento" (como también se ha dicho, vide Carrera de la Red \& Cubo 1991), sino característica en pleno siglo XX o XXI de Canadá y los Estados Unidos, de la India, España, Rusia, Irlanda, Francia o Sudáfrica, como característica lo ha sido igualmente en cualquier otro tiempo, desde la Edad Media a este mismo año 2004...

De hecho, la figura del autor traductor de su propia obra ha estado presente en la historia de este arte y oficio al menos desde los tiempos del historiador judío Flavius Josephus, que escribió en su lengua materna, arameo, los siete libros de su primera obra La guerra de los judíos y años después, en torno al 75 de nuestra era, él mismo la revisó y tradujo al griego, corrigiendo al tiempo alguno de los errores cometidos en el texto primero. En el prefacio a la versión griega ya determina claramente que, "en consideración a los que viven bajo el gobierno de los romanos, me he propuesto traducir a la lengua griega los libros que antes compuse en el idioma de nuestra tierra..." Y lo hizo -añade- porque no podía "sufrir que los griegos y romanos que no estuvieron en aquella guerra ignoren los hechos y no lean [sobre ella] otra cosa que adulaciones e invenciones..." 
Judío también, ya en plena Edad Media, fue Moses Sefardi, natural de Huesca, conocido tras su conversión como Pedro Alfonso (1062-1110), que fuera médico del rey de Aragón Alfonso I y autor de una de las obras más populares de la Edad Media, Disciplina clericalis. Su prólogo concluye con una invocación típica de autotraductor: "Deus igitur in hoc opusculo mihi sit in auxilium, qui me librum hunc componere et in latinum transferre compulit..."

Por la misma época, otro sabio judío, Abraham bar Hiyya (ca. 1065-ca. 1145), residente muchos años en Barcelona, matemático y astrónomo, parece haber compendiado en árabe y luego traducido al hebreo los Fundamentos de la inteligencia y torre de la fe, título de la primera enciclopedia judía sobre matemáticas, astronomía, óptica y música, escrita y traducida a petición de los judíos del sur de Francia, que carecían de textos sobre estas materias. Su ejemplo fue imitado años después, nuevamente del árabe al hebreo, por el judío toledano Juda ben Salomon Cohen, autor en ambas lenguas del tratado enciclopédico La búsqueda de la sabiduría.

En Inglaterra, al obispo de Lincoln Robert Grosseteste (ca. 1168-1253), iniciador de la tradición científica de la Universidad de Oxford y uno de sus primeros cancilleres, se le atribuyen unos Estatuta familiae para el gobierno de casa y hacienda, en tres idiomas: latín, francés e inglés (McEvoy 2000: 147).

Ramon Llull (1232-1316) fue quizá el más prolífico autotraductor de la Edad Media europea. Autor de una obra ingente en catalán, en latín y en árabe (lengua que aprendió ya de adulto con un esclavo moro que acabó suicidándose), escribió precisamente en árabe sus primeras obras, la Lógica de Algacel y el extenso tratado Libro de la contemplación de Dios, que luego él mismo tradujo al catalán y al latín. De esta segunda obra, Llull afirma haber hecho la versión catalana en algo menos de doce meses y haberla concluido en Mallorca en 1272. Al parecer, el propio Llull llevó a cabo una nueva traducción, esta vez al latín, y personalmente entregó un ejemplar en este idioma a la cartuja parisina de Vauvert. Autotraducción al catalán desde una redacción previa en árabe parece haber sido también El libro del gentil. En septiembre del año 1300 Llull completaba en catalán el Libro del ser de Dios, en cuyo prólogo manifiesta su intención de traducirlo al árabe para usarlo en sus controversias con sarracenos, judíos y paganos. Al final de sus días, durante su estancia en Túnez, y quizá debido a su mala vista, Llull contó con la ayuda de fray Simón de Puigcerdá para la versión de hasta quince obras suyas del catalán al latín. De esta última época, por ejemplo, es el Liber de consilio divinarum dignitatum, fechado en Túnez en mayo de 1315, en cuyo colofón latino se afirma que fue inicialmente escrito en árabe, traducido por Llull al romance y de aquí de nuevo al latín.

La versión de obra propia a un segundo idioma parece haber florecido de forma particular en la Península Ibérica durante el siglo XV. Enrique de Villena escribió "en romançe catalan" el Libro de los doze trabajos de Hércules, que "despues trasladolo el mesmo en lengua castellana a suplicaçion de iohan ferrandez de valera el moço su escrivano..." No fue el único. Pocos años después, Alonso de Madrigal, el Tostado, profesor en Salamanca y obispo de Avila, componía primero en latín con traducción posterior al vulgar, a petición de Juan II, un Brevyloquyo de amor e amiçiçia. Y Alonso de Cartagena, obispo de Burgos, seguía su ejemplo con cuatro obras propias, entre ellas una Contemplaçion... ssobre el pssalmo del profeta davit que comiença jusgame dios, que sólo conservamos hoy en versión castellana. 
Algo posteriores en el mismo siglo XV son dos versiones propias latín-castellano de Alfonso de Palencia: la Batalla campal de los perros contra los lobos (1457), una alegoría política de la que también se ha perdido el original latino; y el Tratado de la perfección del triunfo militar (1459), versión hecha por el autor de su anterior De perfectione militaris triumphi; además del prólogo y epílogo bilingües a su Universal Vocabulario. Palencia reconoce que ha traducido sus originales ("este cargo que tengo de romançar lo que yo mesmo conpuse") al ver que "si no se vulgarizase, vendria en conocimiento de pocos, lo qual repugnava a mi deseo". Y como él, Antonio de Nebrija trasladó, "contraponiendo renglon por renglon el romance al latin", el texto de sus Introductiones latinae, un encargo de Isabel la Católica ("vuestra alteza... me mando... que aquellas introduciones de la lengua latina que yo auia publicado \& se leyan por todos vuestros reynos, las boluiesse en lengua castellana contrapuesto al latin el romance..."). Otro tanto hizo con los prólogos bilingües al Lexicon ex sermone latino in hispaniensem y al Dictionarium ex hispaniensi in latinum sermonem.

Del condestable don Pedro de Portugal, que falleciera en Granollers en 1466, conservamos una Satira de infelice e felice vida, texto de juventud, escrita primero en portugués y después traducida por el autor al castellano durante los años de su exilio en Castilla.

Quizá convenga recordar que en los siglos finales del medievo también fueron traductores de su propia obra a un segundo idioma, entre otros muchos, Arnau de Vilanova y Pedro de Luna (papa Benedicto XIII) en España, Nicholas d'Oresme en Francia, Leonardo Bruni en Italia o Marko Marulic (Marcus Marulus) en Croacia.

Los siglos XVI y XVII contemplaron una eclosión sorprendente de la práctica autotraductora, sobre todo entre el latín (lengua mayoritaria de cultura) y los idiomas nacionales. El continente se cubrió así de textos especulares: una obra única en dos lenguas distintas. En Francia tradujeron obra propia del latín (e incluso del griego) al francés, o viceversa: Michel de Boteauville, cura párroco en Guitrencourt, un pueblecito cercano a Nantes, tradujo al francés en 1500 su largo poema latino Carmen de miseriis guerrae Anglorum > Les misères de la guerre. Y Étienne Dolet, el conocido autor de La maniere de bien tradvire d'vne langve en l'avltre, también tradujo al francés en 1538 su poema Francisci Valesii Gallorum Regis Fata, y lo imprimió en 1540 con el título de Les Gestes de Francoys de Valois Roy de France. Un año más tarde compuso otro largo poema en latín, el Genethliacum, para celebrar el nacimiento de su primer hijo; meses después dio a la imprenta su propia traducción al francés, L'Avant-Naissance de Claude Dolet, filtz de Estienne (Worth: 62-68). En el prefacio a esta autotraducción Dolet escribe: "Je me suis bien voulu exercer de le traduire... non pour ostentation de ma rithme, mais pour le proffit que chascun prendra par la traduction d'ung Livre tant plein de doctrine et prudence necessaire à la vie commune..." Y en la misma centuria Louis de Masures; y Jean Dorat, lector de lengua griega en el Collège Royal, traductor al francés de sus propios poemas griegos y latinos; y Rémy Belleau, que en 1573 tradujo al latín sus propios poemas franceses; y Amadis Jamyn, discípulo de Ronsard; y François Moeam, y Bernard du Poey. Y. cómo no, Joachim Du Bellay, con varios poemas autotraducidos del latín al francés, o viceversa, entre ellos el tumulus del "tres chrestien Roy Henri II" y el tumulus del presidente Antoine Minard.

Fuera del ámbito literario ha de mencionarse asimismo a Jean Bodin, uno de los más famosos juristas europeos del siglo XVI, quien en 1576 publicó Les six livres de la 
Republique, un grueso volumen de 759 páginas, y diez años después él mismo lo tradujo al latín: De Republica libri sex.

Y ello sin olvidar a Jean Calvin, que en Ginebra trasladó del latín al francés sucesivas ediciones diferentes de su Christianae religionis Institutio (Basilea 1536, Estrasburgo 1539, etc.). "Il apparait de façon évidente que Calvin a voulu publier aussitôt que possible une traduction française chaque fois qu'une nouvelle édition avait paru..." (Peter 1987: 18). No menos de veintisiete ediciones de esta obra, en latín y en francés, se imprimieron en vida del propio Calvin (ibid.: 18-19), y las traducciones francesas "played an important role not only in the popularization of religious thought in French-speaking lands, but in the development of the French vernacular as a literary vehicle" (Grimm 1969: 316).

En Italia el cardenal Pietro Bembo compuso primero en latín su Historiae Venetae Libri XII, que años más tarde vertió a la lengua vulgar (Storia di Venezia).

En Portugal Pedro Nunes hacía lo propio del portugués al castellano con su Libro de álgebra (1564), "porque a língua Castelhana é mais comum em toda Espanha que a nossa..., e porque nâo careça dela aquela naçâo tanto nossa vizinha, com a qual tanto comunicamos e tanta amizade temos".

En Inglaterra Thomas More traduce al inglés el texto latino de su History of King Richard III. Y John Donne escribe en latín una sátira feroz contra los jesuitas, Conclave Ignatii, para traducirla en el mismo año 1611 al inglés: Ignatius His Conclave. Años más adelante otro tanto hicieron con varias composiciones propias los poetas Abraham Cowley y Andrew Marvell.

En los Países Bajos cabe recordar las versiones propias de Hadrian Damman, Jan van der Noot, Constantijn Huygens, Jacob Cats y sobre todo la figura del filósofo Baruch Spinoza, traductor del latín al holandés, a petición de un círculo de amigos, de su Breve tratado sobre Dios, el Hombre y su Felicidad (1661), del que, perdido el original, sólo se conserva el texto traducido.

En España bastaría con mencionar a Pedro Simón Abril. O mejor a fray Luis de León, que después de componer su primera obra en prosa para la monja Isabel Osorio, la Exposición del Cantar de los Cantares, recibió orden de sus superiores de traducirla al latín. O al jesuita Pedro de Ribadeneira, autor de una Vita Ignatii Loyolae (1572) que traduce luego al castellano como Vida de nuestro Padre Ignacio ("Agora le he traducido y añadido en nuestra lengua castellana para que nuestros hermanos legos de España, otras personas devotas y deseosas de saber los principios de nuestra religion, que no saben la lengua latina, puedan gozar y aprovecharse dél en la suya"). O a otro jesuita, Juan de Mariana, que tras escribir en latín una Historia de España la tradujo al castellano "por el poco conocimiento que de ordinario hoy tienen en España de la lengua latina, además del recelo que tenía no la tradujese alguno poco acertadamente...” En el siglo siguiente, y en Méjico, sor Juana Inés de la Cruz (1651-1695) escribía en latín un epigrama en dísticos elegíacos a la Inmaculada Concepción para después traducirlos a coplas castellanas.

El elenco (siempre incompleto) de traductores de obra propia durante el siglo XVIII incluye, entre otros, al dramaturgo italiano Carlo Goldoni, que en París escribió, en francés, su comedia Le bourru bienfaisant (1771). Dieciocho años después, 1789, él mismo la tradujo al italiano e imprimió el texto italiano también en París, con el título de Il burbero di buen cuore..., traduzione dal francese, fatta dall'autore 
medesimo (Angelini 1993: 299). En la introducción Goldoni admite que en ocasiones ha modificado el original francés para adaptar la obra al gusto del público italiano (ibid.: 284): "Io, padrone dell'opera mia, ho potuto di quando in quando cambiar le frasi, per meglio appropriarle al gusto, e all'uso della mia nazione..."

A lo largo de los siglos XVIII y XIX tradujeron su obra a un segundo idioma el poeta inglés Robert Lloyd y el viajero y lexicógrafo Giuseppe Baretti. Durante su exilio en Francia el dramaturgo español Francisco Martínez de la Rosa escribió en francés el drama Aben Humeya, con una primera representación en París en 1830 en el teatro de La Porte St Martin; ese mismo año publicó el texto en edición bilingüe, francés y español. Joseph Perl y Mendele M. Sforim autotradujeron algunas de sus obras entre hebreo y yiddish. Stéphane Mallarmé vertió al inglés uno de sus poemas, “Le tombeau d'Edgar Poe”. Frédéric Mistral, Premio Nobel en 1904, escribió su largo poema pastoral Mirèio, "avec la traduction littérale en regard", y otro tanto hizo con sus otros poemas, Calendau ("traduction française en regard") y Le poème du Rhone ("texte provençal et traduction française"). Y en Italia Salvatore di Giacomo, en Colombia Samuel Bond y Miguel Antonio Caro, en Alemania Stefan George, en Canadá Honoré Beaugrand (La chasse-galerie, Macloune)...

Por lo que respecta a autotraducciones no literarias, valga poner tres brevísimos ejemplos, de entre decenas de ellos que aquí podrían aducirse: los Elementa Medicinae, traducidos del latín al inglés por el médico escocés John Brown en el siglo XVIII; La clef de la science (1858), de Ebenezer Cobham Brewer, traducida del inglés al francés por su autor ("traduit par l'auteur"); La sociologie criminelle, de Enrico Ferri, "traduction de l'auteur sur la troisième édition italienne".

En el siglo XX y en nuestros propios días autores tan conocidos como Stanislaw Baranczak en Polonia, Talat Sait Halman en Turquía, Vladimir Nabokov y Raymond Federman en los Estados Unidos, D’Annunzio y Ungaretti en Italia, Manuel Puig in Argentina, Vicente Huidobro y nuestro contemporáneo Ariel Dorfman en Chile, los peruanos José María Arguedas y César Moro, el novelista cubano Guillermo Cabrera Infante, Karen Blixen (Isaac Dinesen) en Dinamarca, André Brink y Elsa Joubert en Sudáfrica, Ngûgî wa Thiong'o en Kenya o Rosario Ferré en Puerto Rico..., todos han trasladado obra propia a un idioma distinto del original en que primero la escribieron. Y otro tanto ha ocurrido, y sigue ocurriendo en los Estados Unidos, particularmente entre autores de origen hispano (Sabine Ulibarri, Rolando Hinojosa, Gloria Anzaldúa, Alurista, Tino Villanueva...), en Irlanda, en Paraguay (entre guaraní y español); e igualmente en Canadá, en Pakistán y en la India (Maitreya Devi, Raja Rao, Vijayan...), en Portugal y en Rusia... En resumen, toda una pléyade de escritores contemporáneos de docenas de grupos étnicos, naciones y lenguas.

Tal es el caso, por ejemplo, del novelista Chingiz Aitmatov, escritor en lengua kirghiz, según la UNESCO uno de los novelistas hoy más leídos: "I write my books in Kirghiz and Russian: If a book is first written in Kirghiz, I translate it into Russian, and vice versa..." (Porter 1989: 57). O el del bieloruso Vasil Bykov o Bykaw. O el de Girish Karnad, que en la India de hoy escribe sus obras de teatro en lengua kannada y luego las traduce al inglés. En una entrevista reciente confesaba: "I write my plays in Kannada because I think in Kannada when I write plays. I translate them myself because it is very difficult to translate plays. It's not like translating novels. You have to get the spoken rhythm. You have to know the stage, how a line is breathed..." (En: http:/ /culture.indiainfo. com/literature/interviews/girish_karnad.html). 
Líneas arriba he mencionado a Federico Mistral, premio Nobel en 1904. Como él, también autotraductores, también Premios Nobel de Literatura, Rabindranath Tagore en 1913 (del bengalí al inglés), Samuel Beckett en 1969 (del inglés al francés, y viceversa), Isaac B. Singer en 1978, Czeslaw Milosz en 1980 y Joseph Brodsky en 1987, traductores los tres de obra propia al inglés respectivamente desde el yiddish, el polaco y el ruso.

Tagore parece ser uno de los escasos autores que se han arrepentido de haber traducido su obra a otro idioma. Ya en carta a Edward J. Thompson (Nueva York, febrero de 1921) escribía en alusión a sus autotraducciones: "When I began this career of falsifying my own coins I did it in play; now I am becoming frightened of its enormity and am willing to make a confession of my misdeeds and withdraw into my original vocation as a mere Bengali poet; I hope it is not yet too late to make reparation..." (Dutta \& Robinson 1997: 254). Tres meses después, desde Estocolmo, le confesaba al poeta Thomas S. Moore: "I am convinced that I myself in my translations have done grave injustice to my own work...” (ibid.: 272-273) Y en carta a Amiya Chakravarty, desde Shantiniketan, en febrero de 1937: "It saddens me to drag a poem in Bengali to a foreigh marketplace..." (ibid.: 474).

La universalidad del fenómeno autotraductor se ha extendido asimismo en años recientes a otros muchos campos en los que ahora no pretendo entrar: en 1996, por ejemplo, Verena Jung preparaba su tesis doctoral en la Universidad de Düsseldorf sobre la frecuente autotraducción de textos académicos (lingüísticos, sociológicos, filosóficos) entre inglés y alemán; y Mari Mar Duque García et al. abordaron in 1994 la autotraducción en los muchos ámbitos de la ciencia y la tecnología, que califican de "muy frecuente, debido a que... los científicos se ven obligados a traducir muchos de sus propios artículos al inglés con el fin de que sus trabajos de investigación alcancen una amplia difusión entre la comunidad profesional internacional a la que pertenecen", traducciones estas que sus autores llevan a cabo bien porque desconfían de la fiabilidad de versiones ajenas, bien porque sólo ellos dominan términos y conceptos muchas veces novedosos.

En la ciencia o en la literatura, que lo mismo da, lo cierto es que nadie como el propio autor, nadie con más autoridad que él para resolver las dudas y responder a las preguntas que todo traductor se hace cuando se enfrenta a las sutilezas de un texto ajeno; nadie como él para desentrañar el sentido y el significado cierto del texto. George Steiner (1998: 423) cita la frase que el crítico Samuel Johnson aplicó al poeta inglés John Dryden: "A translator is to be like his author". En nuestro caso, el autor es el traductor, el traductor es el autor.

Permítaseme tratar con detalle dos situaciones actuales, y muy distintas, como son las de España y Francia.

Tierra siempre de fronteras lingüísticas y culturales, en la que han llegado a convivir hasta ocho idiomas distintos, nunca la Península Ibérica había conocido tal proliferación de traducciones de autor como en este último siglo XX. Estrenó la centuria Ramiro de Maeztu, que en 1916 publicó en Londres, y en inglés, su ensayo Authority, Liberty and Function in the Light of the War, y traducido lo reeditó tres años después en Barcelona con el título de La crisis del humanismo. Otro tanto hizo con ensayos y libros propios el trilingüe Salvador de Madariaga. Con todo, el fenómeno de la autotraducción ha resultado particularmente feraz en tierras vascas, gallegas y catalanas. ¿Motivos? Los hay de toda clase y condición, incluso comerciales. 
Autotraductores del vasco al castellano han sido y son la mayor parte de los escritores euskaldunes del siglo XX y de estos primeros años del siglo XXI: Nicolás Ormaechea 'Orixe', Carmelo de Echegaray, Gabriel Aresti o Bernardo Atxaga, que ha traducido al castellano, a veces solo, a veces con colaboradores, buena parte de su obra poética y narrativa, si no toda. Y tras él, un amplísimo elenco de autores más jóvenes, poetas sobre todo, que con frecuencia vierten sus versos a nuevos moldes lingüísticos, entre ellos Koldo Izagirre, Juan Kruz Igerabide y Felipe Juaristi.

Los clásicos de la literatura gallega parecen haber sentido una particular predilección por la traducción propia, desde Eduardo Pondal (1835-1917), Curros Enríquez (1851-1908), Luis Pimentel (1895-1958), Eduardo Blanco-Amor, Luis Seoane o Alvaro Cunqueiro. Muy recientes, de nuestros propios días, son las muchas autotraducciones que del gallego al castellano han hecho Carlos Casares, Alfredo Conde, Carlos G. Reigosa, Suso de Toro o Manuel Rivas, entre decenas de más nombres, poetas y narradores.

No menos numerosos parecen ser los actuales autotraductores catalanes, con una lista que supera con creces el centenar y que, incluída aquí entera, agotaría la paciencia de cualquier lector. Pero no puedo dejar de mencionar entre ellos, siquiera sea a guisa de muestrario, a Pere Gimferrer, Premio Nacional de Poesía y miembro de la Real Academia, Carme Riera, premio Ramón Llull de novela y Anagrama de ensayo, autotraductora de buen número de sus títulos catalanes, Eduardo Mendoza, Antoni Marí o Juan Perucho. Y habría que añadir, entre otros muchos, a Víctor Mora, que tradujo al francés dos de sus relatos, Les platanes de Barcelona y La pluie morte; y a Roser Caminals, autora de un original en inglés, Once Remembered Twice Lived y de su versión catalana, Un segle de prodigis.

El caso de Francia es muy distinto, lingüística y sociológicamente: con una única lengua oficial, pero con un gran número de exiliados (políticos o no) de otros países residentes en su territorio, la autotraducción ha sido y sigue siendo fenómeno frecuente en sus hombres y mujeres de letras. En el siglo XX la lista de autotraductores del francés a otros idiomas, o viceversa, es tan larga que más parece un listín telefónico. Baste recordar unos pocos casos:

El alsaciano Hans Arp, uno de los fundadores del Movimiento Dada en 1916: "Many of Arp's poems exist in parallel French and German versions, and it is often difficult to decide on the face of it which version came first..." (Forster 1970: 82).

Iwan Goll (1891-1950): "His major novels and other prose works are in French; his plays and one of his novels are in German; he frequently translated his plays and his essays -but only rarely his poetry-from one language into another" (ibid.: 80).

Julien Green: buen número de sus textos están en francés y también en inglés: "The Coming of Tango" became "La découverte du tango"; "The Green Lady", "La dame verte"; "An Experiment in English", "Une expérience en anglais"; "Life and Death of a Poet", "Vie et mort d'un poète"; etc. (vide Lucera 1990).

Romain Gary: autotradujo del francés al inglés, a veces con ayuda a ajena, $L a$ danse de Gengis Cohn (1967) > The Dance of Genghis Cohn (1968); y Lady L, escrita en inglés y traducida con el mismo título al francés, Lady L (1963): "J’ai mis six semaines en anglais pour Lady L. et neuf mois pour la version française, cinq ans plus tard..."

Nancy Huston: nacida en Calgary (Canadá), residente durante muchos años en París, escribe bien en francés bien en inglés, y traduce sus propias obras al otro idioma: Plainsong / Cantique des plaines (Canadian Governor General's Award); La 
virevolte, en inglés Slow Emergencies; The Mark of the Angel / L'empreinte de l'ange; Instruments de ténèbres / Instruments of Darkness, una novela que se desarrolla en los actuales Estados Unidos y en la Francia del siglo XVII: Huston escribió en francés los capítulos que transcurren en Francia y en inglés los capítulos americanos, y luego los tradujo al otro idioma.

Piénsese también en los varios escritores rusos que han navegado en la lengua rusa y la francesa a lo largo del último siglo, Elsa Triolet y Jacques Gorbof (Beaujour 1989: 58-61, 241), por ejemplo. Triolet publicó en ruso su primera novela (1925) y cuarenta años más tarde la tradujo al francés: A Tahiti (1964). Tradujo también, del ruso al francés, las primeras páginas de su novela Bonsoir, Thérèse. "A vrai dire escribió- le début de ce premier livre en français, je l'ai quand même écrit en russe, puis traduit..." En 1936 Elsa tradujo, esta vez al ruso, Les cloches de Bâle.

Volvamos al principio.

Richard S. Sylvester dixit: "It is rare enough... for an author to compose a work in one language and then translate it into another". Antoine Berman dixit (1984: 14): "Pour nous, les auto-traductions sont des exceptions". Grady Miller dixit: "Historically, few authors have dared to translate their own works". Christian Balliu dixit: "On en conviendra, les exemples d'autotraduction... sont rarissimes dans le domaine littéraire et ne font qu'exception, comme Nabokov et son Lolita, pour citer un cas".

Hablando de las autotraducciones de Rabindranath Tagore, el crítico indio Sisir Kumar Das escribía hace ahora diez años (1994: 10): "Undoubtedly he is the only major writer in the literary history of any country who decided to translate his own works to reach a larger audience". Me permito subrayar la rotundidad de tres segmentos de esa frase: undoubtedly -the only major writer- of any country.

Visto lo visto, uno no puede menos de preguntarse: ¿saben estos (y otros muchos) críticos de qué están hablando? ¿Puede seguirse hablando de la autotraducción como de un fenómeno 'bastante raro', 'rarísimo' o 'excepcional'? Es cierto que, aisladamente considerado, cada uno de los autores citados puede parecer "un drôle de phenomène", como pueden parecerlo otros muchos no citados, trans-nacionales y/o trans-lingüísticos, y pienso en el alemán Hans Magnus Enzensberger, en el holandés Leo Vroman, en el irlandés Michael Hartnett o en el griego Vassilis Alexakis; o bien, simple y llanamente, en James Joyce, traductor al italiano de Anna Livia Plurabelle: "il lavoro lo fece quasi tutto lui", recordaba en 1945 su colaborador Nino Frank.

Pero sería un gran error seguir pensando y opinando en los términos en que se ha venido haciendo. No estamos ante raras excepciones, sino ante un corpus inmenso, cada vez mayor, de textos traducidos por sus propios creadores. Lejos de ser un 'caso marginal' [border case, borderline case, vide Kálmán 1993), como también se la ha denominado, la traducción de autor cuenta con una larga historia y es hoy en día uno de los fenómenos culturales, lingüísticos y literarios más frecuentes e importantes en nuestra aldea global, y desde luego merecedora de mucha más atención de la que hasta ahora se le ha prestado. 


\section{RÉFÉRENCES}

Angelini, F. (1993): Vita di Goldoni, Roma-Bari, Editori Laterza.

BALliu, C. 2001. "Les traducteurs: ces médecins légistes du texte", Meta, 46/1, p. 92-102.

Beaujour, E. K. (1989): Alien Tongues: Bilingual Russian Writers of the 'First' Emigration, Ithaca and London, Cornell University Press.

Berman, A. (1984): L'Épreuve de l'étranger: Culture et traduction dans l'Allemagne romantique, Paris: Gallimard (edición en inglés: The Experience of the Foreign: Culture and Translation in Romantic Germany, New York, State University of New York Press, 1992, traducción de S. Hayvaert).

Carrera de la Red, A. Et C. R. Cubo (1991): "Fray Luis de León, traductor de sí mismo", Archivo Agustiniano, 75, p. 3-12.

DAs, S. K. (1994): Introduction to The English Writings of Rabindranath Tagore, vol. I: Poems, New Delhi, Sahitya Akademi.

Dutta, K. and A. Robinson (eds.), Selected Letters of Rabindranath Tagore, Cambridge, Cambridge University Press.

Federman, R. (1993): Critifiction: Postmodern Essays, New York, State University of New York Press.

Fiтch, B. T. (1988): Beckett and Babel: An Investigation into the Status of the Bilingual Work, Toronto, Buffalo and London, University of Toronto Press.

Forster, L. (1970): The Poet's Tongues: Multilingualism in Literature, Cambridge, Cambridge University Press in association with the University of Otago Press (New Zealand).

Grimm, H. S. (1969): The Reformation Era, 1500-1650, London, The MacMillan Company.

Kálmán, György C. (1993): “Some Border Cases of Translation”, in José Lambert and André Lefevere (eds.), Translation in the Development of Literatures: Proceedings fo the XIth Congress of the International Comparative Literature Association, Bern: Peter Lang and Leuven, Leuven University Press, p. 69-72.

LuCERA, G. (1990): Introducción a La langue et son double, en el vol. VI de las Oeuvres complètes de Green, París: Gallimard (Bibliotèque de la Pléiade).

McEvoy, J. (2000): Robert Grosseteste, Oxford, Oxford University Press.

Miller, G. (1999): “The Author as Translator”, ATA Spanish Language Division: Selected SpanishRelated Presentations, St. Louis, Missouri, ATA 40th Annual Conference, p. 11-17.

Peter, R. (1987): «La première édition de l'Institution de la religion chrétienne de Calvin», in Rodolphe Peter et Bernard Roussel (eds.), Le Livre et la Réforme, Bordeaux, Société des Bibliophiles de Guyenne, p. 117-134.

Popovic, A. (1976): Dictionary for the Analysis of Literary Translation, Edmonton, Department of Comparative Literature, The University of Alberta.

Porter, R. (1989): Four Contemporary Russian Writers, Oxford, Berg Publishers.

Shuttleworth, M. and M. Cowie (1997): Dictionary of Translation Studies, Manchester, UK, St Jerome Publishing.

Steiner, G. (1998) [1975]: After Babel: Aspects of Language \& Translation, Oxford, Oxford University Press.

Sylvester, R. S. (ed.) (1963): The Complete Works of St. Thomas More. Vol. 2. The History of King Richard III, New Haven, CT and London, Yale University Press [introduction to Vol. 2].

Tanqueiro, H. (2000): "Self-Translation as an Extreme Case of the Author-Translator-Dialectic", in A. Beeby et al. (eds.), Investigating Translation: Selected Papers from the 4th International Congress on Translation (Barcelona 1998), Amsterdam-Philadelphia, John Benjamins.

Wechsler, R. (1998): Performing Without a Stage: The Art of Literary Translation, North Haven, Connecticut, Catbird Press.

Whyte, Christopher (2002): “Against Self-Translation”, Translation \& Literature, vol. 11, Part 1 (Spring 2002).

Worth, V. (1988): Practising Translation in Renaissance France: The Example of Etienne Dolet, Oxford, Clarendon Press. 